\title{
A NOVEL MAGNETOMETER EMPLOYING MAGNETOVISCOUS EFFECT OF FERROFLUIDS
}

\author{
G. Hatipoglu ${ }^{l}$, and S. Tadigadapa ${ }^{I^{*}}$ \\ Department of Electrical Engineering, \\ Pennsylvania State University, University Park, USA
}

\begin{abstract}
In this work, we utilize ferrofluids deposited atop an ultrahigh permeability Metglas ${ }^{\circledR}\left(\mathrm{Fe}_{85} \mathrm{~B}_{5} \mathrm{Si}_{10}\right)$ amorphous thin film coated micromachined quartz resonator ( $-\mathrm{QCR}$ ) array as a unique magnetometer introduced by our group for the first time to the best of our knowledge. The magnetometer employs magnetoviscous effect of ferrofluids i.e. external magnetic field induced variations in the viscosity of a ferrofluid emulsion. The -QCR array detects these viscosity changes by virtue of its high sensitivity to viscoelastic changes in adsorbed films on its electrode. Initial measurements on the device has shown promising results, where it was possible to detect magnetic field inputs as low as $\sim 60 \mathrm{nT}$ with a SNR of 3:1.
\end{abstract}

\section{INTRODUCTION}

The scalar magnetometer introduced in this work, utilizing a highly sensitive quartz -viscometer coated with a high permeability material, tracks the viscosity changes within the ferrofluid as a sensor mechanism to detect and quantify the magnetic field strength.

Ferrofluids, which are emulsions of nano/ micro ferroparticles in a carrier liquid demonstrate highly ordered microscopic configurations under the influence of external magnetic fields [1]. The phenomenon of viscosity change of ferrofluids arising due to applied magnetic field is defined as the magnetoviscous effect [2]. The emulsion undergoes various complex structural transitions upon the application of an external magnetic field, whereby, an ensemble of Brownian magnetic nano-particles in the emulsion spontaneously organizes into columnar structures due to magnetic field induced dipole interactions [3]. The ordered chain-like columnar structures as a result of the applied magnetic field follow a hexagonal structural pattern. The length of the agglomerates and the separation distance between them are very sensitive to the small magnitude changes of the applied magnetic field as well as the frequency of the magnetic field [4].

On the other hand, the magnetization curve of an ensemble of such nano-ferroparticles is hysteresis-free. This has important consequences, as suspended superparamagnetic particles can be constantly manipulated within the fluid matrix using a magnetic field, and they do not agglomerate after the removal of the field. The particles have enough thermal energy and they are stabilized by the surfactants of about $2 \mathrm{~nm}$ thickness, which aids to recover from an aggregated state preventing further permanent aggregation. Hence, it is easy to switch on and off the magnetic interaction in the ferrofluid. The properties of the ferroluid (Ferrotec Inc, EMG 911) studied here are listed in Table 1.

Acoustic wave sensing techniques based on quartz crystal resonators $(\mathrm{QCR})$, in principle, are able to characterize not only the physical properties of the layer, but also viscoelastic properties of the layer. A typical thickness shear mode quartz crystal resonator

Table 1: Ferrofluid properties.

\begin{tabular}{|c|c|c|c|c|}
\hline $\begin{array}{c}\text { Particle } \\
\text { diameter }\end{array}$ & $\begin{array}{c}\text { Saturation } \\
\text { magnetization }\end{array}$ & $\begin{array}{c}\text { Magnetic } \\
\text { Particle Conc. }\end{array}$ & $\begin{array}{c}\text { Density } \\
\boldsymbol{a}_{\mathbf{2 5}}{ }^{\circ} \mathbf{C}\end{array}$ & $\begin{array}{c}\text { Viscosity } \\
\boldsymbol{(} \mathbf{2 7} 7^{\circ} \mathbf{C}\end{array}$ \\
\hline $10 \mathrm{~nm}$ & $\begin{array}{c}11 \mathrm{mT} \\
( \pm 10 \%)\end{array}$ & $2 \mathrm{vol} \%$ & $\begin{array}{c}890 \\
\mathrm{~kg} / \mathrm{m}^{3}\end{array}$ & $2.0 \mathrm{mPa}-\mathrm{s}$ \\
\hline
\end{tabular}

consists of a thin slice of single-crystal, piezoelectric quartz, with very large lateral dimensions in comparison to its thickness, which is sandwiched between two metal electrodes. Application of a sinusoidal electric field using the electrodes sets up a shear wave through the thickness of the quartz [5]. At resonance, the equivalent electrical circuit of the QCM is modeled as consisting of a capacitor $C_{0}$ in parallel with the motional arm of the circuit consisting of a series circuit of $L_{m}, C_{m}$, and $R_{m}$ and is commonly referred to as the Butterworth Van Dyke (BVD) model. The exceptionally low phase noise characteristic exhibited by quartz resonators allows for a precision of few parts in $10^{14}$ and is the primary reason for their widespread use in frequency control applications. The phase noise is quantitatively specified in terms of the $Q$-factor defined as the half width at the full maximum of the resonance curve or in terms of an energy dissipation factor $D$, which is inversely proportional to the decay time constant and is also equal to the $1 / Q$-factor. The resonance frequency and the $Q$ factor of the resonance are significantly affected by any surface load placed on the quartz crystal. Thus, QCRs are widely used as sensors to determine the properties of deposited layers on top such as surface mass density and recently the viscoelastic properties [6].

The QCM frequency shift resulting from the deposition of viscoelastic layer in a viscous liquid ambient can be analyzed using a continuum mechanics approach [7]. In order to model this situation, the QCM surface is considered to be in intimate contact with a layer of continuous viscoelastic slab with an infinitely thick Newtonian liquid overlayer on one of its surfaces. Under the assumption that the thickness of the bulk liquid layer is much larger than the decay length of the acoustic wave in the liquid, analytical expressions for the frequency, $Q$-factor, and dissipation factor changes with respect to liquid loading conditions can be derived [8]. Our previous work has demonstrated that the high frequency micromachined quartz resonators are especially suited for monitoring small changes in the viscoelastic loading in films adsorbed on their surface. Figure 1 compares the dependence of the frequency and $Q$-factor shift as a function of the viscosity and elastic modulus of a $35 \mathrm{~nm}$ thick soft polymer like film adsorbed on a commercial $5 \mathrm{MHz}$ and micromachined $65 \mathrm{MHz}$ resonators. It is clear that miniaturized resonators provide a greater resolution of these properties. Water-like liquid overlayer is assumed in these graphs. The miniaturized resonators provide a greater resolution for these properties. A large variation in Q-factor and frequency for small changes in the viscoelastic properties of the adsorbed films is highly desirable for accurate real-time monitoring of viscoelastic changes of the ferrofluids.

From the BVD model, the electrical admittance $Y$ of the resonator can be simply written as

$Y=G+j B=j B_{0}+Y_{m}$

where $B_{0}=\omega C_{0} ; Y_{m}=\frac{1}{R_{m}+j X_{m}} ; X_{m}=\omega L_{m}-\frac{1}{\omega C_{m}}$

$G$ and $B$ are the real and imaginary parts of admittance respectively, $B_{0}$ is the admittance of the parallel (static) arm, and $Y_{m}$ is the admittance of the motional arm consisting of series connected $R_{m}, L_{m}$ and $C_{m}$ the motional resistance, inductance and capacitance respectively. From this equation, it is possible to write 

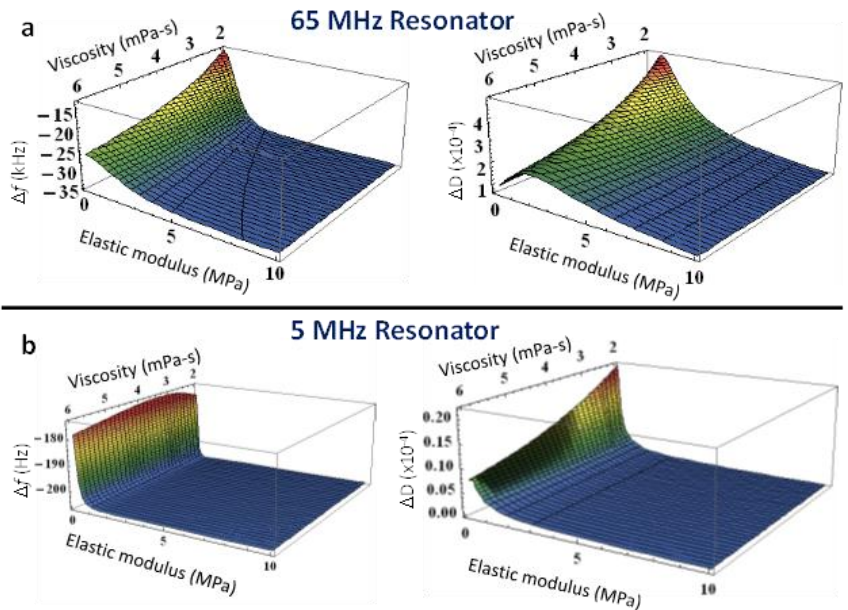

Figure 1: Comparison of the frequency and dissipation factor sensitivity of (a) $65 \mathrm{MHz}$ and (b) $5 \mathrm{MHz}$ resonators to variations in the viscosity and modulus of the adsorbed films. (Film thickness $=$ $35 \mathrm{~nm}$ and density $=1040 \mathrm{~kg} / \mathrm{m}^{3}$ have been assumed here). Clearly, the $65 \mathrm{MHz}$ resonator has a better resolution over wider range of values of these properties.

the following parametric representation is possible

$$
\left(G-\frac{1}{2 R m}\right)^{2}+\left(B-B_{0}\right)^{2}=\left(\frac{1}{2 R_{m}}\right)^{2}
$$

Thus tracking the peak of the real part of the admittance allows for a direct measurement of the motional resistance or realtime tracking of the damping effect on the quartz resonator arising due to the changing viscosity of the ferrofluid due to applied magnetic field. In this work the -QCR array electrical characteristics, affected by the magnetoviscous effect in ferrofluids, were quantitatively measured by the real-time tracking of the maximum point on the admittance curve, which in turn enables to monitor and quantify the magnetic field strength changes in real-time. In order to increase the sensitivity of the magnetometer, the -QCR is coated with an ultra-high permeability Metglas ${ }^{\circledR}\left(\mathrm{Fe}_{85} \mathrm{~B}_{5} \mathrm{Si}_{10}\right)$ amorphous thin film. Thin film of Metglas ${ }^{\circledR}$ acts as a flux concentrator [9], which is explained and discussed in the following sections in detail.

\section{THE PRINCIPLES OF MAGNETOMETER OPERATION}

\section{Device Configuration}

The -QCR array used in this work is composed of 8 resonators with each having $500 \mathrm{~m}$ diameter circular electrode. The quartz thickness in between the electrodes is $\sim 25 \mathrm{~m}$, which was etched to this final thickness from a $\sim 100 \mathrm{~m}$ thick AT-cut quartz substrate using reactive ion etching in the $\mathrm{SF}_{6}$ and Argon plasma. In this work only one pixel was utilized for the magnetometer tests, although the other pixels may be used to determine the direction of the magnetic field in future experiments. Figure 2(a) shows 3-D schematic rendering of one pixel of the array with ferrofluid loading. The device is packaged into a modified dual-in-line ceramic package with a $5 \times 5 \mathrm{~mm}$ reservoir for the ferrofluid loading. Figure 2(b) depicts the cross sectional view of the pixel with the packaging.

A cylindrical Neodymium magnet having a diameter larger than the resonator electrode area is placed in close proximity to the resonator such that the magnet provides a uniform DC magnetic bias in $+Y$-direction. This magnetic field coalesces the nanoparticles in the ferrofluid to form ordered agglomerate atop the resonator electrode, which can then be treated as a viscoleastic layer. A weak-to-moderate bias introduces some degree of columnar aggregation and orientation within the ferrofluid. It is found and experimentally shown that the low level of orientation enhances the sensitivity of the device due to enhanced magnetoviscous effect. Once a particular level of aggregation exists, the viscosity changes due to magnetic field strength are sharper and more evident [2].

The application of a periodic magnetic field in $+\mathrm{X}$ direction (in the plane of the quartz resonator electrode as shown in Fig. 2.(b) to the device, results in continuous perturbation in the ordering arrangement of the ferrofluid aggregate and therefore induces a continuous change in the viscoelastic response of the quartz resonator admittance maximum. In addition, the applied magnetic field can change the separation distance between ordered structures alter and thus the packing density could also be altered [4]. The authors postulate that the viscosity change within the fluid is mainly a function of this separation distance, which is highly sensitive to low amplitude changes in the applied magnetic field as well as its frequency [4].
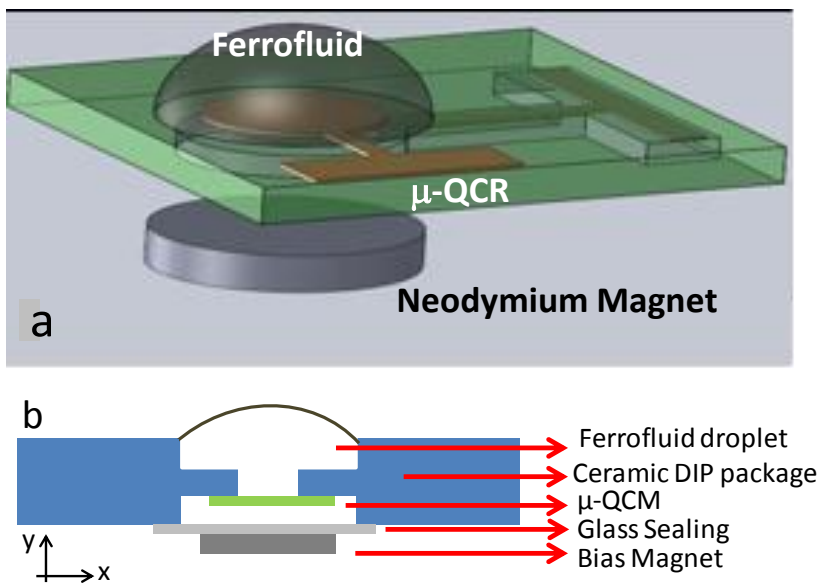

Figure 2: a) The magnetometer configuration (cross sectional view). b) $3 D$ schematic showing the resonator, magnet,and ferrofluid drop.

Figure 3 shows the experimental setup above. The Helmholtz coil has a diameter of $15 \mathrm{~cm}$ and 140 turns $(\mathrm{N}=140)$. The magnetic field is precisely controlled via the current. The $\mu$-QCR admittance characteristics were monitored in real-time using an Agilent 4294A impedance analyzer. The admittance maxima of the device was tracked and recorded as a function of the applied magnetic field.

\section{Metglas as a Flux Concentrator}

The working electrode, the top electrode in Fig. 2(a) is coated with $20 \mathrm{~nm} \mathrm{Ti} / 100 \mathrm{~nm}$ of Metglas ${ }^{\mathbb{B}}$ via ion beam deposition. The resonator area is lithographically patterned and delineated using lift-off process. Metglas ${ }^{\circledR}$ is an amorphous alloy, consisting of $\mathrm{Fe}_{85} \mathrm{~B}_{5} \mathrm{Si}_{10}$ and has a very high relative magnetic permeability $(r)$ of 45000 . This property of Metglas ${ }^{\circledR}$ is utilized to concentrate the low amplitude magnetic flux vectors into the thin film of Metglas ${ }^{\circledR}$.

Figure 4(a) shows a 2D COMSOL magnetostatics model demonstrating the flux concentration effect. A thin strip of 


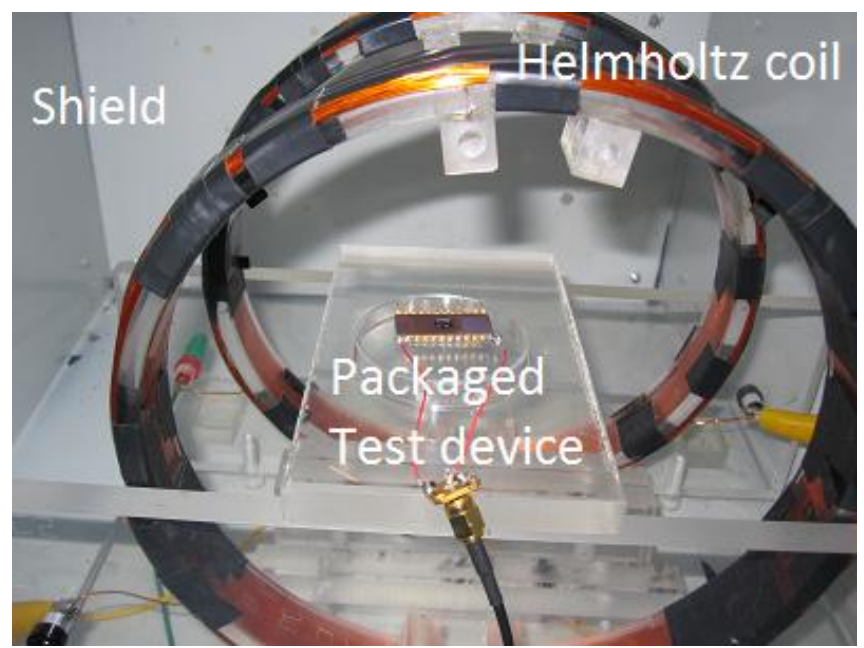

Figure 3: The Experimental Setup showing the shield, the packaged test device in DIP ceramic package and the Helmholtz coil. The -QCR array without the ferrofluid atop is unresponsive to magnetic field.

$100 \mathrm{~nm} \mathrm{Metglas}{ }^{\circledR}$ is assumed to be surrounded by air. The concentration effect is analyzed without the DC bias magnetic field from the neodymium magnet under the QCR in order to focus on the concentration effect in $+X$-direction, which is the direction of the applied magnetic field. From the left boundary of the domain to the right boundary of the domain, a uniform magnetic flux of $\sim 100 \mathrm{nT}$ is applied. As it is observed, the flux lines start to bend at the regions closer to Metglas ${ }^{\circledR}$ due to its high $r$. Moreover, the flux lines start to concentrate gradually within the length of the thin strip, reaching to a maximum in the mid section. This is shown in Fig. 4(b) where, the magnitude of the magnetic flux along the strip is plotted. The maximum peak flux that is $\sim 375 \mathrm{~T}$ corresponds to the middle point $(\mathrm{X}=1.5 \mathrm{~mm})$ of the $100 \mathrm{~nm}$ thick Metglas $^{\circledR}$ film. The flux magnitude sharply decreases to the applied uniform field that is $100 \mathrm{nT}$ at the outer regions of the Metglas ${ }^{\circledR}$ film at far ends which is $0.5 \mathrm{~mm}$ away from left and right sides of the film. Therefore, $100 \mathrm{nT}$ of the applied magnetic flux is concentrated within the Metglas ${ }^{\circledR}$ thin film to a maximum value of $\sim 375 \mathrm{~T}$, corresponding to a three orders of magnitude of enhancement. This flux concentration and enhancement aids to amplify the small magnitude changes of the applied field and therefore the sensitivity of the device. Therefore, the ferroparticles within the ferrofluid respond better to amplified magnetic field and agglomeration/deglomeration happen more effectively. Consequently, the quantification of the weak magnetic field via the

-QCR becomes more sensitive and feasible through the concentration effect.

\section{RESULTS \& DISCUSSION}

As it is emphasized above, the device is characterized by applying a square wave of magnetic field in $+\mathrm{X}$-direction (see Fig. 2(b)) through the Helmholtz coil. The characterization focuses on nano-Tesla region. The device response is tracked for randomly selected magnetic field square inputs (50\% duty cycle) of $720 \mathrm{nT}$, $360 \mathrm{nT}, 180 \mathrm{nT}, 130 \mathrm{nT}$ and $60 \mathrm{nT}$. Figure 5 shows the device response for selected magnetic fields of $720 \mathrm{nT}, 360 \mathrm{nT}$ and $60 \mathrm{nT}$ for a single on/off cycle. As the magnetic field is switched on, the admittance peak increases spontaneously to its maximum amplitude. When the field is off, the admittance peak decreases to its minimum value. A possible explanation for this admittance shift
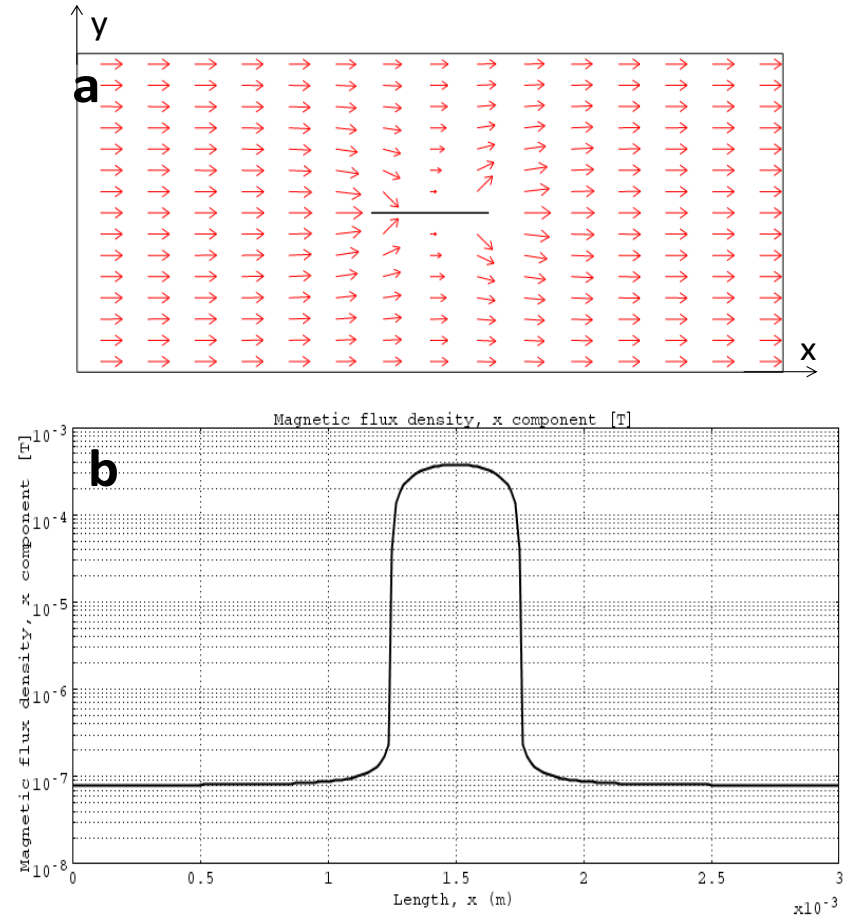

Figure 4: (a) The interaction of the magnetic flux lines with high relative permeability Metglas ${ }^{\circledR}$ thin film. A uniform field of 100 $n T$ is applied in $+x$ direction. The total length of the domain is 3 $\mathrm{mm}$ (b) The concentration effect is maximum in the middle (at $x=1.5 \mathrm{~mm}$ ) of the thin strip. The flux is concentrated about 3 orders of magnitude.

is the decreasing viscosity of the ferrofluid emulsion upon the application of the magnetic field, which perturbs the ordered state from the DC bias field. It can be thought to arise due to the increased interaction of the ferrofluid particles leading to a more tightly bound state in the presence of the magnetic field. This could arise either due to the spontaneous arrangement of the ferrofluid particles into an ordered structure or through tighter interaction leading to a more rigid network. When the magnetic field is switched off, the ferrofluid particles rapidly lose the ordered state due to their thermal energy thereby increasing the viscosity of the ferrofluid. That is why the role of the DC magnetic bias field is very important in this experiment and depending on its strength can change the response of the ferrofluid to the incident magnetic field.

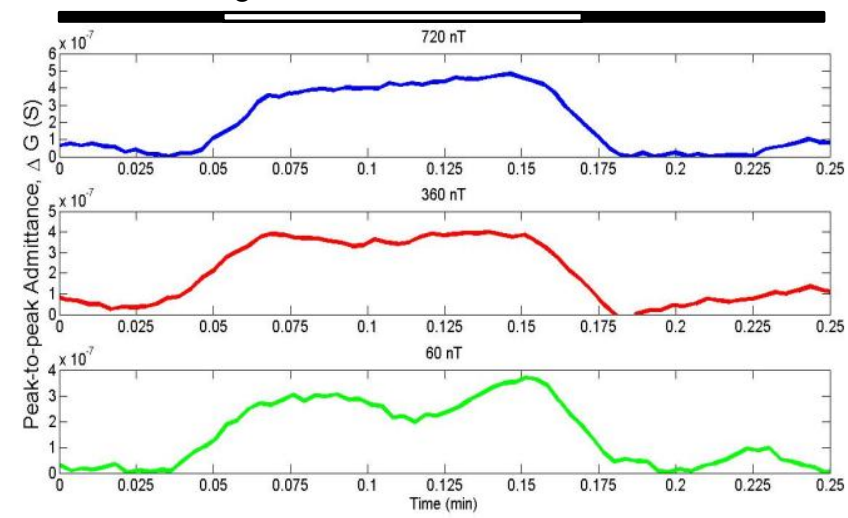

Figure 5: Maximum admittance vs time. As the magnetic field is switched on (indicated with the white bar on top), the viscosity is decreased and admittance increases. 


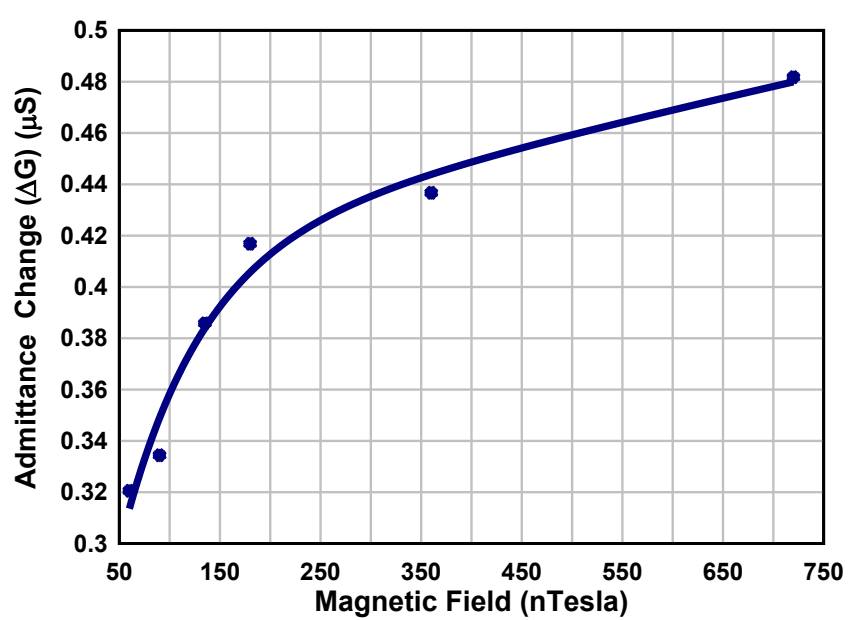

Figure 6: Peak-to-peak admittance for different square wave magnetic field amplitudes. A smooth exponential fit has been used.

The device was further characterized at the selected magnetic fields by quantifying the peak-to-peak change in resonator admittance, $\Delta \mathrm{G}$, obtained from Fig. 5. A single value is chosen for on and off values using averaging. The peak-to-peak change in resonator admittance versus the magnetic field is plotted in figure 6 . The resonator shows a non-linear response. For the experimental conditions used, the admittance change due to magnetic field amplitude showed a sharper slope between $60 \mathrm{nT}$ and $200 \mathrm{nT}$ than at higher fields.

Despite the fact that the lowest magnetic field amplitude that has been detected is $60 \mathrm{nT}$ with a SNR of $\sim 3: 1$ with the current experimental setup and facilities, the magnetometer described here has the potential for sub-nanoTesla resolutions at room temperatures. Nevertheless, the questions relating to the linearity of response, the optimal bias magnetic field (magnitude and orientation), and the ultimate sensitivity of the sensor need to be further investigated. The other pixels can be utilized to determine the direction of the magnetic field in future studies.

\section{CONCLUSION}

In this work, a novel scalar magnetometer is proposed. The magnetometer utilizes: (i) ferrofluids and their mangetoviscous properties as the sensing mechanism, (ii) the highly sensitive QCR to detect and quantify the magnetoviscous effects, (iii) the flux concentration effect to amplify the weak magnetic field via the deposition of an ultra-high permeability thin film on working electrode, and (iv) real time magnetic field measurement by tracking the peak admittance. This study and device configuration can be also considered as a new study of the high frequency perturbation characteristics of ferrofluids.

\section{ACKNOWLEDGEMENT}

This work was supported in part by the U.S. Army Research Office under Grant W911NF-07-1-0327 and in part by the National Science Foundation (NSF) under Grant ECCS 0925438. The use of facilities at the PSU Site of the NSF National Nanotechnology Infrastructure Network (NNIN) under Agreement 0335765 is acknowledged. The authors thank to Feng Li for sputtering Metglas on the -QCR. GH acknowledges partial support from Scientific and Technical Research Council of Turkey (TUBITAK) for a 2213-International PhD. Fellowship Program.

\section{REFERENCES}

[1] V. S. Mendelev and A. O. Ivanov, "Ferrofluid aggregation in chains under the influence of a magnetic field," Physical Review E, vol. 70, p. 051502, 2004.

[2] S. Odenbach and S. Thurm, "Magnetoviscous Effects in Ferrofluids." vol. 594, S. Odenbach, Ed., ed: Springer Berlin / Heidelberg, 2003, pp. 185-201.

[3] M. Ivey, et al., "Magnetic-field-induced structural transitions in a ferrofluid emulsion," Physical Review E, vol. 63, p. 011403, 2000.

[4] C.-Y. Hong, et al., "Evidence of multiple states of ordered structures and a phase transition in magnetic fluid films under perpendicular magnetic fields," Applied Physics Letters, vol. 75, pp. 2196-2198, 1999.

[5] S. J. Martin, et al., "Characterization of a quartz crystal microbalance with simultaneous mass and liquid loading," Analytical Chemistry, vol. 63, pp. 2272-2281, 1991/10/01 1991.

[6] P. Kao, et al., "Human Serum Albumin Adsorption Study on 62-MHz Miniaturized Quartz Gravimetric Sensors," Analytical Chemistry, vol. 80, pp. 5930-5936, 2008/08/01 2008.

[7] M. V. Voinova, et al., "Viscoelastic Acoustic Response of Layered Polymer Films at Fluid-Solid Interfaces: Continuum Mechanics Approach," Physica Scripta, vol. 59, p. 391, 1999.

[8] P. Kao, et al., "Fabrication and performance characteristics of high-frequency micromachined bulk acoustic wave quartz resonator arrays," Measurement Science and Technology, vol. 20, p. 124007, 2009.

[9] Z. Fang, et al., "Enhancing the magnetoelectric response of Metglas/polyvinylidene fluoride laminates by exploiting the flux concentration effect," Applied Physics Letters, vol. 95, p. 112903, 2009.

\section{CONTACT}

*S. Tadigadapa, Tel: +1-814-865-2739; sat10@.psu.edu 